\title{
Psychological well-being among college students: A study from Central India
}

\author{
Arvind Sharma1, Tej Pratap Singh², Richa Sharma ${ }^{3}$, Jagmohan Singh Dhakar4, \\ Aditi Bharti ${ }^{5}$ \\ ${ }^{1}$ Professor, ${ }^{2,5}$ Postgraduate Resident, ${ }^{4}$ Statistician cum Tutor, Department of Community Medicine, ${ }^{3}$ Medical Officer, \\ Department of Emergency Medicine, Netaji Subhash Chandra Bose Medical College, Jabalpur, Madhya Pradesh, India
}

Background: Psychological well-being (PWB) is affected by age, physical health, personality, life experiences, socioeconomic status, and culture. Age is an important social determinant of health and age-based analysis is necessary to rectify one's health and health care. Aims and Objectives: This study aims to assess the PWB of college students and to determine the correlation between age and PWB aspects. Materials and Methods: This cross-sectional study was conducted among 210 college students selected by simple random sampling. Ryff's PWB scale, a 42-item version used for the assessment of PWB, and data were collected by self-administering questionnaires. Statistical Analysis: Internal consistency of the scale was estimated and descriptive statistics, independent samples t-test, and regression analysis were applied. Results: The mean age of the participants was $19.41 \pm 1.69$ years and $61 \%$ were male. Cronbach's alpha coefficient for internal consistency of Ryff's PWB scale was 0.814 in this study. The mean of PWB was $205.42 \pm 11.04$ and $\geq 20$ years scored higher in all the parameters of PWB than $<20$ years, differences were $(t=3.62, P=0.001)$ significant. Simple linear regression showed that age was a highly significant $(P=0.002)$ predictor of PWB. Conclusion: The study illustrated that students of 20 years or more had better PWB in terms of all dimensions than $<20$ years-, and also suggested the need for future research, like, to deepen on the conceptual and relations between age and PWB, and to analyze the impact that social changes have toward age group on PWB.

Key words: College students; Correlation; Psychological well-being; Simple linear regression analysis; Undergraduate students

\section{INTRODUCTION}

Psychological well-being (PWB) is defined as a multidimensional psychological state with positive functioning, contentment, and absence of mental illnesses. An individual's perception of himself changes overtime, becoming more related to temporary differences as he grows older and less related to interpersonal comparisons..$^{1-3}$ PWB is beneficial for adults to live a completely healthy life, and making it an important aspect of one's life in the younger age groups. ${ }^{4}$ College life may be hectic and full of psychological stress regularly. According to Chao (2012), among college age groups, stress has increased tremendously over the past decade. ${ }^{5}$ It is, therefore, important to understand factors that impact PWB on the college studying cohort. All these perceptions are taken into consideration for the comprehension of PWB. Hence, the experience an individual acquires during one's lifetime can change the ideals to which he aspires and the way he assesses his well-being.

Young people perceive themselves as making significant progress in their adolescence and have great expectations for the future so the scores in their self-assessments for the dimensions of purpose in life and personal growth are higher. ${ }^{6}$ One of the ways to get closer to understanding the concept of PWB is to study the factors associated with it.

According to Ryff, PWB refers to the extent to which people feel that they have meaningful control over their 
life and their activities. ${ }^{7}$ Nevertheless, PWB problems have become increasingly common among college students nowadays who are prone to psychological problems. ${ }^{8}$ Researchers have shown that poor PWB appears to be a very crucial and critical issue among college students. Nevertheless, for any of these stages or transitions, students need to deal with intellectual, social, and psychological transformations, as these transitions are also perceived as a process of maturation. Therefore, students often have to deal with challenges and problems that hinder the completion of their studies. ${ }^{9-11}$ Moreover, they need to avoid any high stress which is becoming increasingly worrying, as it has been found that the number of psychological problems and their severity are on the rise among the student population. ${ }^{12-14}$ Hence, problems such as financial restrain and time constraints, which limit the time left to complete their assignment and research papers due to work, classes, and family affect student's PWB. ${ }^{10,15}$ Poor PWB and mental state may affect the positive learning and task performance of the students.

\section{Aims and objectives}

This study aims to assess the psychological well-being of college students and to determine the correlation between age and PWB aspects.

\section{MATERIALS AND METHODS}

\section{Ethical permission}

The study was approved by the institutional ethics committee with Approval No. IEC/2021/4995). The procedures followed were in accordance with the ethical standards of the responsible committee on human experimentation (institutional or regional) and with the Helsinki Declaration of 1975, as revised in 2000.

\section{Study design, study population, and sampling}

This cross-sectional study was carried out among college undergraduate students in Jabalpur of Central India. A total of 210 students been selected by simple random sampling method. The data were collected by self-administering the questionnaire to the students which consisted of two parts. The first part recorded the demographic information including the participant's age and gender. The second part was the Ryff's scale, the 42-item version used for the assessment of PWB.,16 Ryff had introduced six core dimensions of PWB, specifically: (1) Self-acceptance (state of having positive thoughts and feelings about oneself); (2) positive relations with others (ability to engage in a warm and trusting relationship with others); (3) autonomy (ability to be independent and coping with social pressure); (4) environmental mastery (ability to adapt, change, or create one's environment according to one's needs through physical and mental activities); (5) purpose in life (state of having objectives and goals in life and working toward achieving goal oriented), and (6) personal growth (continuously growing and developing as oneself). Number of items in each subscale of Ryff's PWB scale was 7. Seven items of each Ryff's subscale are divided between positive and negative items. On a 42-item version scale, 20 PWB items were positively worded and 22 items negatively worded. Negatively worded items scores were reversed before conducting the analysis so that high values indicated well-being. ${ }^{7}$ The scores for six subscales were calculated as averages; higher scores mean greater PWB. For each of 42 items, the responses to the questions were based on a 7-point scale ranging from 1 to 7: Strongly disagree (1), slightly disagree (2), disagree (3), neutral (4), agree (5), slightly agree (6), and strongly agree (7). ${ }^{7,17,18}$

The purpose of the study was explained before administering the questionnaire to the students, privacy was assured, and informed consent was taken from all the students. Those students who were not willing to participate in study were excluded from the study. No students were pressurized and it was emphasized to choose the answer which they actually felt, and students were asked not to put their personal information on questionnaires. Permissions were obtained from the heads of the respective colleges.

\section{Statistical analysis}

Data were analyzed using the Statistical Package for the Social Sciences (IBM SPSS Statistics 20) software (version 20.0). To assess the internal consistency of the Ryff's PWBS, Cronbach's alpha $(\alpha)$ coefficients were calculated and descriptive statistics were analyzed. The relation between PWB and age group was determined by Pearson's correlation coefficient and simple linear regression analysis for the prediction of PWB. $\mathrm{P}<0.05$ will be considered as statistically significant.

\section{RESULTS}

A total of 210 undergraduate college students participated in the study to assess the levels of PWB and 197 questionnaires were analyzed, 13 forms were rejected because of being incompletely filled. The age of the students ranged from 17 to 25 years with the mean age of students is 19.41 ( \pm 1.69$)$. Among the study subjects, majority of participants were $<20$ years $(62.4 \%)$ and remaining were above and/or equal to 20 years ( $37.6 \%)$, and out of the 197 students, there were $61 \%$ of male and $39 \%$ of female. In this study, Cronbach's alpha $(\alpha)$ coefficient for measure of internal consistency reliability of scale was 0.814 , indicating good internal consistency. 
Descriptive analysis was used to determine the level and pattern of students' PWB on six of its dimensions (autonomy, environmental mastery, personal growth, positive relations with other, purpose in life, and selfacceptance). The findings indicated high score on the dimension of purpose in life $(\mathrm{M}=35.82, \mathrm{SD}=3.88)$, followed by personal growth $(\mathrm{M}=35.74, \mathrm{SD}=3.86)$, positive relations with others $(\mathrm{M}=34.3, \mathrm{SD}=4.01)$, selfacceptance $(M=33.61, S D=4.00)$, autonomy $(M=33.36$, $\mathrm{SD}=4.36)$, and environmental mastery $(\mathrm{M}=32.59$, $\mathrm{SD}=4.02)$ in Table 1.

Table 2 displays the difference between student's age groups and their level of PWB. The mean score of PWB among $<20$ years age group is $202.11(\mathrm{SD} \pm 17.23)$ and $\geq 20$ years is 210.91 ( $\mathrm{SD} \pm 15.21)$. The differences of PWB and age group were found to be significant in PWB, autonomy, environmental mastery, personal growth, positive-relation, purpose in life, and self-acceptance.

Table 3 represents the correlation between age and PWB, it was found that age was significantly positively correlated to PWB $\left(r_{s}=0.221, P=0.01\right)$. This indicates as the age increases, the level of PWB increases. Similar findings were observed in the subdimensions of PWB, which

\begin{tabular}{|c|c|c|c|}
\hline & Mean & Std. deviation & $\mathbf{N}$ \\
\hline Age & 19.41 & 1.693 & 197 \\
\hline PWB & 205.42 & 17.010 & 197 \\
\hline Autonomy & 33.36 & 4.362 & 197 \\
\hline Environmental mastery & 32.59 & 4.020 & 197 \\
\hline Personal growth & 35.74 & 3.860 & 197 \\
\hline Positive relations & 34.30 & 4.011 & 197 \\
\hline Purpose in life & 35.82 & 3.881 & 197 \\
\hline Self-acceptance & 33.61 & 4.007 & 197 \\
\hline
\end{tabular}

shows significant association with autonomy $\left(\mathrm{r}_{\mathrm{s}}=0.185\right.$, $\mathrm{P}=0.01)$, positive relation $\left(\mathrm{r}_{\mathrm{s}}=0.157, \mathrm{P}<0.05\right)$, purpose in life $\left(\mathrm{r}_{\mathrm{s}}=0.157, \mathrm{P}<0.05\right)$, and self-acceptance $\left(\mathrm{r}_{\mathrm{s}}=0.235\right.$, $\mathrm{P}=0.01)$.

Table 4 represents the model summary indicating correlation between age and PWB among students was found to be $R=0.221$, further $R$ square change $=0.049$ which represents the actual contribution of age to PWB, the real covariance magnitude of predictor variable: Age which contributes to the criterion variable: PWB came out as $4.9 \%$. Similarly, age contributes to various dimensions of PWB among which self-acceptance came out to be maximum, that is, $5.55 \%$ and minimum was personal growth, that is, $0.7 \%$. It also indicates that age (predictor) influences PWB (criterion). The statistical value given in the mentioned table indicates that ANNOVA test was applied and $\mathrm{F}=10.056$ values are significant for age and also show the positive relationship between age and PWB. The value of beta is $r=0.221$ which indicates that age significantly influences degree of PWB among students. The relationships between these two variables represent linear correlation among students; it means that when age increases PWB also increases and vice-versa. Similar kind of linear correlation is among other dimensions of PWB such as autonomy $(\mathrm{F}=6.903$, value of beta is $\mathrm{r}=0.221)$, positive relations $(\mathrm{F}=4.934$, value of beta is $\mathrm{r}=1.157)$, purpose in life $(F=4.931$, value of beta is $r=0.157)$, and selfacceptance $(\mathrm{F}=11.359$, value of beta is $\mathrm{r}=0.235)$ and these dimensions also significantly influence degree of PWB among students.

\section{DISCUSSION}

The main aim of this study was to investigate the influence of age group on the college student's PWB conducted

\begin{tabular}{|c|c|c|c|c|c|c|}
\hline Variables & Age group in year & Mean & SD & SEM & $t$ value & $P$ value \\
\hline \multirow[t]{2}{*}{ PWB } & $<20$ & 202.11 & 17.238 & 1.554 & 3.620 & 0.000 \\
\hline & $20-25$ & 210.91 & 15.211 & 1.768 & & \\
\hline \multirow[t]{2}{*}{ Autonomy } & $<20$ & 32.59 & 4.435 & 0.400 & 3.236 & 0.001 \\
\hline & $20-25$ & 34.62 & 3.915 & 0.459 & & \\
\hline \multirow[t]{2}{*}{ Environmental mastery } & $<20$ & 32.26 & 4.063 & 0.366 & 1.484 & 0.139 \\
\hline & $20-25$ & 33.14 & 3.915 & 0.455 & & \\
\hline \multirow{2}{*}{ Personal growth } & $<20$ & 35.31 & 3.890 & 0.351 & 2.018 & 0.045 \\
\hline & $20-25$ & 36.45 & 3.728 & 0.433 & & \\
\hline \multirow{2}{*}{ Positive relations } & $<20$ & 33.66 & 4.149 & 0.374 & 2.948 & 0.004 \\
\hline & $20-25$ & 35.36 & 3.548 & 0.412 & & \\
\hline \multirow[t]{2}{*}{ Purpose in life } & $<20$ & 35.40 & 3.989 & 0.360 & 1.991 & 0.048 \\
\hline & $20-25$ & 36.53 & 3.612 & 0.420 & & \\
\hline \multirow[t]{2}{*}{ Self-acceptance } & $<20$ & 32.89 & 4.248 & 0.383 & 3.334 & 0.001 \\
\hline & $20-25$ & 34.81 & 3.259 & 0.379 & & \\
\hline
\end{tabular}




\begin{tabular}{|c|c|c|c|c|c|c|c|c|}
\hline & Age & PWB & Aut & Env & Grow & Relat & Purp & Acc \\
\hline Age & 1 & & & & & & & \\
\hline PWB & $0.221^{* *}$ & 1 & & & & & & \\
\hline Aut & $0.185^{\star *}$ & $0.722^{\star *}$ & 1 & & & & & \\
\hline Env & 0.116 & $0.671^{\star *}$ & $0.355^{\star *}$ & 1 & & & & \\
\hline Grow & 0.081 & $0.701^{* *}$ & $0.425^{\star *}$ & $0.360^{* *}$ & 1 & & & \\
\hline Relat & $0.157^{*}$ & $0.650^{* *}$ & $0.364^{* *}$ & $0.311^{\text {** }}$ & $0.325^{\star *}$ & 1 & & \\
\hline Purp & $0.157^{*}$ & $0.720^{* *}$ & $0.374^{* *}$ & $0.339^{* *}$ & $0.453^{\star *}$ & $0.403^{* *}$ & 1 & \\
\hline Acc & $0.235^{\star *}$ & $0.763^{* *}$ & $0.483^{* *}$ & $0.470^{* *}$ & $0.423^{* *}$ & $0.348^{* *}$ & $0.501^{* *}$ & 1 \\
\hline
\end{tabular}

PWB: Psychological well-being g; aut, autonomy; env, environmental mastery; grow, personal growth; relat, positive relations; purp, purpose in life; acc, self-acceptance. **Correlation is significant at the 0.01 level (2-tailed). * Correlation is significant at the 0.05 level (2-tailed).

Table 4: Summary of simple linear regression analysis for the prediction of PWB of students $(n=197)$

\begin{tabular}{lccccccc}
\hline Dependent variables & $\mathbf{R}$ & $\mathbf{R}^{2}$ & $\mathbf{B}$ & SEB & Beta & F & Sig. \\
\hline PWB & 0.221 & 0.049 & 2.226 & 0.702 & 0.221 & 10.056 & 0.002 \\
Autonomy & 0.185 & 0.034 & 0.477 & 0.181 & 0.185 & 6.903 & 0.009 \\
Environmental mastery & 0.116 & 0.013 & 0.276 & 0.169 & 0.116 & 2.666 & 0.104 \\
Personal growth & 0.081 & 0.007 & 0.185 & 0.163 & 0.081 & 1.298 & 0.256 \\
Positive relations & 1.157 & 0.025 & 0.372 & 0.168 & 0.157 & 4.934 & 0.027 \\
Purpose in life & 0.157 & 0.025 & 0.360 & 0.162 & 0.157 & 4.931 & 0.028 \\
Self-acceptance & 0.235 & 0.055 & 0.555 & 0.165 & 0.234 & 11.359 & 0.001 \\
\hline
\end{tabular}

Predictors: Age, PWB: Psychological well-being, B: Unstandardized coefficients, SEB: Standard error of B. Independent variable as age while dependent variable as PWB and dimensions of PWB

in Central India. As analyzed and reported, the results concluded that the level of PWB was on an extreme level among college students. Nevertheless, the dimension with the highest mean score was purpose in life, followed by positive relations, self-acceptance, autonomy, and environmental mastery. In this study context, college students perpetually deal with challenges. It could be explained as in college students, is more vulnerable to get into psychological pressure due to various reasons such as physical development, age differences, identity crisis, emotionalism, and fear of new responsibility or failure in obtaining jobs. ${ }^{17,19}$

This study explained that PWB in the age group of $\geq 20$ years was better than in $<20$ years and showed a statistically significant difference with PWB in this particular age group. With a similar finding, one study revealed that age had a powerful role in predicting PWB. ${ }^{6,7,18,20}$ Significant correlations were found between autonomy and age among the college students, although, contrary to this, a study by Ryff, Ryff and Keyes, and Chen and Persson who stressed that autonomy is higher in middle-aged and old age group. ${ }^{6,7,18,21,22}$ According to the finding of Ryff, autonomy is a character of individualistic cultures. However, Chirkov indicated that either individualistic or collectivistic practices might be enacted more or less autonomously, demonstrating that autonomy as an attribute of behavior regulation is different from individualism/collectivism. ${ }^{23}$ In this study, it might be concluded that educated people may be aware of their potential to develop their autonomy.
In this study, it is important to note that there was no significant correlation between environmental mastery and age among the college students in this study. Based on the results of the previous studies, older adults would score higher than young adults on environmental mastery., ${ }^{722}$ Similarly, no significant correlation was also observed between personal growth and age in this study, this is in contradiction with the past studies which confirmed, young adults would score higher than older adults on personal growth. ${ }^{6,718}$ In addition, there was a significant correlation between the positive relationship with others and age among students in this study. This finding was also concluded by a previous study that indicated the positive relationship with others and age. ${ }^{24}$

Meanwhile, a significant correlation was found among the graduate students in this study in terms of purpose in life and age. This finding is in alignment with several researchers such as Ryff (1989), Ryff (1991), Ryff and Keyes (1995), and Chen and Persson (2002). ${ }^{67,18,22}$ Purpose in life acts as a compass, guiding young people's lives in positive directions. As a motivator, it orients life goals and daily decisions by directing the use of personal resources such as time, energy, and effort toward pro-social aims. ${ }^{25-27}$ Students will give relevance to schoolwork and personal meaningfulness only after they identify a purpose in life and connect that purpose to their academic experience, that it may otherwise lack. Another study stated that purpose in life can serve as an important source of motivation, and students lacking it do not fully achieve their intentions, engagements, and contributions in academic life particularly and life span in general. ${ }^{27}$ There was a significant correlation 
discovered between self-acceptance and age among the graduate students in this study. This result is in line with the findings of the previous studies, which showed age stability for this dimension..$^{6,728}$

\section{Limitations of study}

Some limitations of present study should be noted. First, the present study finding are based on cross-sectional design and thus limits the ability to determine casual relationship. Second limitation of this study is that it relies on Ryff's scale of psychological well-being which is selfreported scale. As with self-report instruments, students may respond in ways that are socially desirable rather than reveal their actual response to each statement.

\section{CONCLUSION}

The results of the present study illustrated that student of 20 years or more had better PWB in terms of all dimensions rather than $<20$ years, and also suggested some issues for future research, for example, to deepen on the conceptual and relations between age and PWB, to analyze the role of the cultural rules and standards on age differences in PWB, and to analyze the impact that social changes have toward age group on the PWB.

\section{ACKNOWLEDGMENT}

The authors take this opportunity to thank the Dean NSCB Medical College, Jabalpur for wholehearted support for this study, the college principles for giving the permission to conduct the study, and all the students who participated in this study.

\section{REFERENCES}

1. Sinha JN and Verma J. Social Support as a Moderator of the Relationship between Alcoholism and Psychological Well-Being, Social and Applied Issues; 1992.

2. Khanbani M, Asghar $A$ and Parvar MG. Examining the relationship between gender and psychological well-being. J Sociol Res. 2014;5(1):53-58.

https://doi.org/10.5296/jsr.v5i1.5436

3. Sharma G. Effect of demographic variables on psychological well-being and quality of life. Int J Soc Sci Humanit Res. 2014;2(3):290-298.

4. Molina-García J, Castillo I and Queralt A. Leisure-time physical activity and psychological well-being in university students. Psychol Rep. 2011;109(2):453-460.

https://doi.org/10.2466/06.10.13.pr0.109.5.453-460

5. Chao RC. Managing perceived stress among college students: The roles of social support and dysfunctional coping. J Coll Couns. 2012;15(1):5-21.

https://doi.org/10.1002/j.2161-1882.2012.00002.x
6. Ryff CD. Possible selves in adulthood and old age: A tale of shifting horizons. Psychol Aging. 1991;6(2):286-295.

https://doi.org/10.1037/0882-7974.6.2.286

7. Ryff CD. Happiness is everything, or is it? Explorations on the meaning of psychological well-being. J Personal Soc Psychol. 1989;57(6):1069-1081.

https://doi.org/10.1037/0022-3514.57.6.1069

8. Yang YT. Stress, coping, and Psychological Well-Being: Comparison among American and Asian International Graduate Students from Taiwan, China, and South Korea; 2010.

9. Lovitts BE. Being a good course taker is not enough: A theoretical perspective on the transition to independent research. Stud High Educ. 2005;30(2):137-154. https://doi.org/10.1080/03075070500043093

10. Abiddin NZ and Ismail A. Attrition and completion issues in postgraduate studies for student development. Int Rev Soc Sci Humanit. 2011;1(1):15-29.

11. Lessing $A$ and Schulze S. Postgraduate supervision and academic support: Students' perceptions. S Afr J High Educ. 2002;16(2):139-149.

12. Sherina $M$, Rampal $L$ and Kaneson N. Psychological stress among undergraduate medical students. Med J Malaysia. 2004;59(2):207-211.

13. Regehr C, Glancy D and Pitts A. Interventions to reduce stress in university students: A review and meta-analysis. J Affect Dis. 2013;148(1):1-11.

https://doi.org/10.1016/j.jad.2012.11.026

14. Byrd DR and McKinney KJ. Individual, interpersonal, and institutional level factors associated with the mental health of college students. J Am Coll Health. 2012;60(3):185-193. https://doi.org/10.1080/07448481.2011.584334

15. Lange $C$ and Byrd $M$. The relationship between perceptions of financial distress and feelings of psychological well-being in New Zealand University students. Int J Adolesc Youth. 1998;7(3):193-209.

https://doi.org/10.1080/02673843.1998.9747824

16. Abbott RA, Ploubidis GB, Huppert FA, Kuh D, Wadsworth ME and Croudace TJ. Psychometric evaluation and predictive validity of Ryff's psychological well-being items in a UK birth cohort sample of women. Health Qual Life Outcomes. 2006;4:76

https://doi.org/10.1186/1477-7525-4-76

17. Sharma A and Sharma R. Internet addiction and psychological well-being among college students: A cross-sectional study from Central India. J Family Med Prim Care. 2018;7(1):147-151. https://doi.org/10.4103/jfmpc.jfmpc_189_17

18. Ryff $C D$ and Keyes CL. The structure of psychological wellbeing revisited. J Pers Soc Psychol. 1995;69(4):719-727.

19. Green SB, Salkind NJ and Akey TM. Using SPSS for Windows: Analyzing and Understanding Data. New York: Prentice Hall; 1997.

20. Punia $\mathbf{N}$ and Malaviya R. Psychological well being of first year college students. India J Educ Stud. 2015;2(1):60-68.

21. Roslan S, Ahmad NA, Nabilla NA and Ghiami Z. Psychological well-being among postgraduate students. Acta Med Bulgarica. 2017;44:35-41.

https://doi.org/10.1515/amb-2017-0006

22. Chen $Y$ and Persson $A$. Internet use among young and older adults: Relation to psychological well-being. Educ Gerontol. 2002;28(9):731-744. 
https://doi.org/10.1080/03601270290099921

23. Chirkov VI. Culture, personal autonomy and individualism: Their relationships and implications for personal growth and wellbeing. In: Zheng G, Leung K and Adair JG, editors. Perspectives and Progress in Contemporary Cross-Cultural Psychology. Beijing: Light Industry Press; 2007. p. 247-263.

24. Ryff CD, Magee WJ, Kling KC and Wing EH. Forging macromicro linkages in the study of psychological well-being. In: Ryff CD and Marshall VM, editors. The Self and Society in Aging Processes. New York: Springer; 1999. p. 247-278.

25. Damon W, Menon J and Bronk KC. The development of purpose during adolescence. Appl Dev Sci. 2003;7(3):119-128.

26. Benson PL. All kids are Our Kids: What Communities Must do to Raise Caring and Responsible Children and Adolescents. Stanford: Jossey-Bass; 2006.

https://doi.org/10.3200/tchs.81.5.237-238

27. Damon W. The Path to Purpose: Helping Children find Their Calling in Life. New York: Free Press; 2008.

28. Ryff CD. Challenges and opportunities at the interface of aging, personality, and wellbeing. In: John OP, Robins RW and Pervin LA, editors. Handbook of Personality: Theory and Research. New York: Guilford; 2008. p. 399-418.

Authors Contribution:

AS- Concept and design of the study, prepared first draft of manuscript, data acquisition; manuscript editing; TPS- Interpreted the results; reviewed the literature and manuscript preparation; RS- Data acquisition and revision of the manuscript; JSD- Statistical analysis and interpretation; and AB- Manuscript editing, manuscript review.

\section{Work attributed to:}

Netaji Subhash Chandra Bose Medical College, Jabalpur - 482 003, Madhya Pradesh, India.

Orcid ID:

Dr. Arvind Sharma - (1) https://orcid.org/0000-0003-4567-6920

Dr. Tej Pratap Singh - (1) https://orcid.org/0000-0002-4005-7357

Dr. Richa Sharma - (D) https://orcid.org/0000-0001-8376-648X

Mr. Jagmohan Singh Dhakar - (D https://orcid.org/0000-0001-9592-3227

Dr. Aditi Bharti - (1) https://orcid.org/0000-0001-9745-6482

Source of Funding: None, Conflicts of Interest: None. 